\title{
Fractal Based Analysis, Modeling and Prediction of Neurophysiological Time Series
}

\author{
Hamidreza Namazi \\ School of Mechanical and Aerospace Engineering,Nanyang Technological University,Singapore \\ hnamazi@ntu.edu.sg
}

\begin{abstract}
Neurophysiology is a branch of physiology and neuroscience that is concerned with the study of the functioning of the nervous system. Neurophysiological time series are signal shaped time series which are governed from different processes that are related to nervous system. The most important neurophysiological time series which is directly related to the activity of the nervous system is the EEG signal. Besides EEG time series there are some other neurophysiological signals which are indirectly related to the activity of the nervous system. For instance, the respiratory signal is another type of neurophysiological time series which is governed from the process of the nervous system. In fact, ventilation occurs under the control of the autonomic nervous system from the medulla oblongata and the pons in the brainstem. These areas of the brain form the respiration regulatory center, a series of interconnected brain cells within the lower and middle brain stem which coordinate respiratory movements. We can call other types of neurophysiological time series such as fixational eye movements time series, etc.
\end{abstract}

Analysis, modeling and prediction of neurophysiological time series always have been an important issue between researchers. One of the most important aspects of these modeling and analysis has been from mathematical point of view. In fact, using mathematics for the modeling purpose helps scientists to investigate precisely about the processes. Employing mathematical models can help them in forecasting the processes in the next step where the biological modeling cannot help.

Fractals are scale-invariant geometric objects. A scale invariant object can be self-similar or selfaffine. A self-similar object is a union of rescaled copies of itself which is isotropic or uniform in all directions. But in case of self-affine objects, the mechanism is an isotropic or dependent on the direction. Regular fractals have higher self-similarity, but random fractals have a weaker selfsimilarity.

The class of regular fractals includes many familiar simple objects, such as line intervals, solid squares, and solid cubes, and also many irregular objects. The scaling rules are characterized by "scaling exponents" (dimension). "Simple" regular fractals have integer scaling dimensions. Complex self-similar objects have non-integer dimension. Therefore, it is completely incorrect to define fractals as geometric objects having "fractional" (non-integer) dimension. Fractals maybe defined as geometric objects whose scaling exponent (dimension) satisfies the Szpilrajn inequality:

$$
\aleph \geq D_{T}
$$

where $\boldsymbol{N}$ is the scaling exponent (dimension) of the object and $D_{T}$ is its topological dimension, i.e., Euclidean dimension of units from which the fractal object is built. For example, in case of Brownian motion: the path of a particle, a line of dimension one, traveling for a long time over a plane region, eventually covers the entire plane, an entity of dimension two. In case of multi fractal system a single fractal dimension cannot describe its dynamics. In this case, a continuous spectrum of exponents is needed.

We deal with many multi fractal systems in nature such as fully developed turbulence, heartbeat dynamics, etc. In general, fractal analysis can be categorized in two parts. Fractal pattern analysis and fractal time series analysis. Scientists have found that many of neurophysiological time series are multi fractals which are described by a spectrum of fractal dimensions. So, fractal theory can be used for analysis, modeling and prediction of neurophysiological time series. In all analysis, modeling and prediction of time series using fractal theory, the concept of fractal Brownian motion comes to the account where unlike classical Brownian motion, the increments of time series need not be independent. 
During years different works have been reported in literatures which focused on analysis of neurophysiological time series using fractal theories [1-10]. In a recent work on prediction of epileptic seizure onset we proposed a new methodology which is based on studying the EEG signals using two measures, the Hurst exponent and fractal dimension. In order to validate the proposed method, it was applied to EEG signals of patients with epilepsy by computing the Hurst exponent and fractal dimension in pre-seizure, seizure, and some post seizure activities, and then the results were validated versus the reference data. We found out that there are big fluctuations in the Hurst exponent and fractal dimension plots of EEG time series before the seizure onset. The results of these analyses showed that were able to forecast the onset of a seizure on the average of 25.76 seconds before the time of occurrence [11].

In case of employing fractals for the modeling purpose limited works have been reported in literatures. We can call the work which model the human EEG signal upon receiving external stimuli, based on fractional diffusion equations. In this model first we developed fractional diffusion equation by relating the diffusion of external stimulus to the brain and then applied this model to the EEG signal as the feature of human consciousness. This model was solved and generated a time series. In order to check the similarity between the generated time series and the real EEG signals we computed the Hurst exponent for both time series. The results showed that the Hurst exponent spectra for the modeled EEG signal mimic the Hurst exponent spectra for the real EEG signal. The results of this modeling showed very good agreement with the real human EEG signal [12]. Please note that modeling of neurophysiological time series using fractals is not limited to fractional diffusion models and can be done using other types of fractal equations (models), such as fractal telegraph equations, based on the problems in hand.

Another aspect of using fractals is predictability. In the last mentioned paper we brought the predictability to fractional diffusion model by inserting the Hurst exponent. In fact, the Hurst exponent as indicator of long term memory of the process brings the predictability to the account. Based on the value of the Hurst exponent in each point of time series the process can be Brownian motion, positively or negatively correlated. Fractal dimension $(D)$ and the Hurst exponent $(H)$ have relationship only when the time series is long. This relation can be expressed as:

$$
D=2-H
$$

So, modeling of fractal time series can lead to their prediction by considering the long term memory through incorporating the Hurst exponent to the models.

In overall, using fractal theory can help us in analysis, modeling and prediction of neurophysiological time series. In fact, it will have outstanding clinical advantages which can speed up clinical practice for treatment of patients and drug design development.

Beside all efforts done in using fractal theory in analysis, modeling and prediction of neurophysiological time series, still a lot of works are remain to do in this area. So, there are many open windows to researchers in order to analyze, model and forecast the neurophysiological time series.

\section{REFERENCES}

[1] C.-K. Peng, Joseph E. Mietus, Yanhui Liu, Christine Lee, Jeffrey M. Hausdorff, H. Eugene Stanley, Ary L. Goldberger, and Lewis A. Lipsitz. Quantifying Fractal Dynamics of Human Respiration: Age and Gender Effects. Annals of Biomedical Engineering, Vol. 30, pp. 683-692, 2002.

[2] Szeto, H.H., Cheng, P.Y., Decena, J.A., Cheng, Y.,Wu, D.L., Dwyer, G., 1992. Fractal properties in fetal breathing dynamics. Am. J. Physiol. 263, R141-R147.

[3] Larsen PD, Elder DE, Tzeng YC, Campbell AJ, Galletly DC. Fractal characteristics of breath to breath timing in sleeping infants. Respir Physiol Neurobiol. 2004 Feb 25;139(3):263-70.

[4] Quan Wang, Elizabeth Kim, Katarzyna Chawarska, Brian Scassellati, Steven Zucker, Frederick Shic. On Relationships Between Fixation Identification Algorithms and Fractal Box Counting Methods. ETRA 2014, March 26 - 28, 2014, Safety Harbor, Florida, USA.

[5] Wu DW, Anderson NC, Bischof WF, Kingstone A. Temporal dynamics of eye movements are related to differences in scene complexity and clutter. J Vis. 2014 Aug 11;14(9). pii: 8. doi: 10.1167/14.9.8. 
[6] Lee JS, Spiegel D, Kim SB, Lee JH, Kim SI, Yang BH, Choi JH, Kho YC, Nam JH. Fractal analysis of EEG in hypnosis and its relationship with hypnotizability. Int J Clin Exp Hypn. 2007 Jan;55(1):14-31.

[7] Zorick T, Mandelkern MA (2013) Multifractal Detrended Fluctuation Analysis of Human EEG: Preliminary Investigation and Comparison with the Wavelet Transform Modulus Maxima Technique. PLoS ONE 8(7): e68360. doi:10.1371/journal.pone.0068360

[8] Zappasodi F, Olejarczyk E, Marzetti L, Assenza G, Pizzella V, et al. (2014) Fractal Dimension of EEG Activity Senses Neuronal Impairment in Acute Stroke. PLoS ONE 9(6): e100199. doi:10.1371/journal.pone.0100199.

[9] Carlos Gómez, Ángela Mediavilla, Roberto Hornero, Daniel Abásolo, Alberto Fernández. Use of the Higuchi's fractal dimension for the analysis of MEG recordings from Alzheimer's disease patients. Medical Engineering \& Physics 31 (2009) 306-313

[10] Haruhiko Nishimura, Isao Nakagiri, Yuko Mizuno-Matsumoto, Ryouhei Ishii, Satoshi Ukai, Kazuhiro Shinosaki. Time-series fractal analysis of MEG changes induced by emotional stimulation. Journal of Japan Society for Fuzzy Theory and Intelligent Informatics Vol. 20 (2008) No. 1 P 117-128.

[11] Namazi H, Kulish VV, Hussaini J, Hussaini J, Delaviz A, Delaviz F, Habibi S, Ramezanpoor S. A Signal processing based analysis and prediction of seizure onset in patients with epilepsy. Oncotarget. 2015 Nov 17. doi: 10.18632/oncotarget.6341.

[12] Hamidreza Namazi and Vladimir V. Kulish, "Fractional Diffusion Based Modelling and Prediction of Human Brain Response to External Stimuli," Computational and Mathematical Methods in Medicine, vol. 2015, Article ID 148534, 11 pages, 2015. doi:10.1155/2015/148534 